Bernt-Peter Robra and Stefan Felder*

\title{
Homburg's Lockdown Analysis: Conclusions without Data and an Appropriate Estimation Model
}

https://doi.org/10.1515/ev-2020-0015

Received May 30, 2020; accepted June 24, 2020

\begin{abstract}
This is a critical response to Homburg (2020) who claimed that lockdowns were superfluous and ineffective. We demonstrate that Homburg's database is not sufficient to support his claim and that his econometric approach not suitable for an analysis of the lockdowns' additional effect. However, we corroborate Homburg's observation that the number of infections was already receding when the German lockdown came into force.
\end{abstract}

Keywords: corona, SARS-CoV-2, covid-19, lockdown

JEL Classification: I18

\section{Introduction}

Homburg (2020) attempts to study the effectiveness of a comprehensive lockdown. It becomes clear in his discussion that he does not refer to traditional containment measures such as social distancing, testing, contact tracing or quarantine. Rather, he "questions [.] that the lockdowns of entire economies yielded additional benefits". With regard to Germany, he refers to the restrictions of March 23, 2020, the so-called contact ban and the countrywide closure of restaurants and services in body care.

With his short, but far-reaching conclusion "lockdowns were superfluous and ineffective”, Stefan Homburg achieved broad public attention. An evaluation of contact restrictions in Germany and elsewhere is of interest in retrospect, but also for the scope of future containments of the SARS-CoV-2 epidemic and the

*Corresponding author: Stefan Felder, Faculty of Business and Economics, University of Basel, Peter Merian-Weg 6, 4002 Basel, Switzerland, e-mail: stefan.felder@unibas.ch

Bernt-Peter Robra: Otto-von-Guericke University Magdeburg, Magdeburg, Germany 
prevention of a second wave. For this reason, it appears to be appropriate to examine the data and the derivation of Homburg's conclusion.

\section{Data Base and Method}

The basic idea of Homburg's analysis is straightforward. For the evaluation of the SARS-CoV-2 containment measures, the evolution of the daily number of new infections is crucial. However, daily reported cases do not provide a clear picture of the epidemic's dynamics since they depend on the intensity of the quest for cases (test criteria, intensity of testing, diligence of contact identification) and, for that reason, are intertwined with the control intensity. Daily reported Covid-19 deaths, by contrast, as conjectured by Homburg, "are much more reliable" than diagnoses. Thus, the time series of daily Covid-19 deaths is his basis of the evaluation of epidemic control, using Johns Hopkins University's (JHU) database covering the period between March 1 and April 13, 2020.

From the evolution of the daily Covid-19 deaths, the unobserved ("true") evolution of daily new infections can be deduced if the average time interval between infection and death is known and constant. Homburg applies an estimate of 23 days from two respectable publications of case series from China. With this interval, he transfers the evolution of deaths back in time to reconstruct the evolution of infections. Homburg's stylized Figure 1 shows both curves: in black the curve of deaths, and to the left, strikingly red colored, the curve of new infections. Naturally, the red curve does not include any independent information. The corresponding text reads „the red bars correspond to the actual infections, which are not directly observable“, bringing home to the reader the unobservable.

As the progression and the peak of infections and deaths are identical except for the time shift, the robustness of data interpretation solely depends on how trustworthy daily mortality data are. Homburg finds their maximum for April 7 in calendar week 15; minus roughly three weeks lead time points to March 15. March 12 marks the day of the crisis conference between chancellor Merkel and the prime ministers of the German federal states (Merkel: "Where possible, abstain from social contacts”). Therefore, effective, although not yet specified determinants shortly after March 12 should have resulted in a reversal of the unobserved trend of new infections, as inferred from the observable Covid-19 mortality.

\subsection{Quality of Certified Causes of Death}

The quality of mortality statistics is subject to a long and broad critical literature. In Germany as, elsewhere, the classification of Covid-19 deaths has been discussed in 
terms of death due to or with coronavirus infection. Arguably, classification errors are approximately constant over time, so that the peak of the mortality trajectory can still be interpreted. However, the number of deceased with Covid-19, who were positively tested and counted as epidemic deaths, is confounded with case ascertainment and test intensity, factors from which Homburg wants to abstract. In contrast, there must have been patients who died at home or in a skilled nursing facility due to Covid-19, but did not count as epidemic deaths when related symptoms were not recognized, not scrutinized by tests or individuals were not conspicuous within contact chains. Total (or all cause) mortality is resistant to misclassification, so we inspect it in Section 3.

\subsection{Time Interval Between Infection and Death}

Homburg's quoted sources report a 95\% confidence interval of the incubation period ranging from 4.5 to 5.8 days (Lauer et al. 2020) and the time between onset of symptoms and death from 16.9 to 19.2 days (Verity et al. 2020). A confidence interval of one or two days around the 23 day-interval would not fundamentally qualify the relevant finding that the dynamics of the infections must have changed around mid-March. However, the mechanism of epidemic control remains a black box. Loose couplings limit a causal inference on the effect of the regulatory intervention. People might have changed their contact behavior even earlier, on their own initiative or in anticipation of containment measures. This is very likely, given the results of the COSMO survey: already in the first wave (March 3 and 4), 96\% of the respondents knew the mode of SARS-CoV-2 transmission (droplets), 95\% stated hand hygiene and 90\% sneezing hygiene (Betsch et al. 2020). In any case, deaths are not a perfect correlate of epidemic dynamics. In this respect, the author's premises need to be qualified, even though they are not necessarily false.

\subsection{Replication of Homburg's Logistic Curve - and Beyond}

Homburg assumes that the epidemic follows a logistic function $f(t)=\frac{S}{1+a e^{-b t}}$, with $t$ as time and $a, b$ and $S$ as functional parameters. Below, the saturation limit $S$ is of particular interest. The symmetry of the distribution of new infections allows him to derive the turning point $t^{*}$ where new infections reach their maximum $\left(t^{*}=-(\ln (1 / a)) / b\right.$ and the parameters $a$ and $b$ are estimated separately for each country). He does not present any details of his computations. Using the publicly available JHU data, we reverse engineer his approach using the software package $\mathrm{R}$ 
for model fitting and visualizations. Applying non-linear least squares via the nls function from base R, we minimize RSS $=\sum_{i=1}^{T}\left(t_{i}-f\left(t_{i}, \Theta\right)\right)^{2}$, with $\Theta=\left(S, t^{*}, c\right)$ and $f\left(t_{i}, \Theta\right)=\frac{S}{1+e^{\left(t^{*}-t_{i}\right) / c}}$. In this parametrization, slightly different from Homburg's, the turning point $t^{*}$, conveniently, is a parameter of the curve.

Figures 1 and 2 show the fitted curves for the evolution of cumulated and daily deaths for Germany and Italy, respectively, based on data for the period from $t_{1}=$ March 1 to $t_{44}=$ April 13, 2020 (Figure 2 corresponds to Homburg's Figure 2 for Italy, except that it also includes observations until April 30 in order to assess the robustness of his fitted model). The respective estimated mortality rate at the end of the epidemic is $0.005 \%$ for Germany and $0.036 \%$ for Italy. These rates are obtained as ratios of the implied saturation levels (parameter $S$ of the logistic curve) and population totals; they agree (in the case of Italy up to minor rounding) with Homburg's figures.
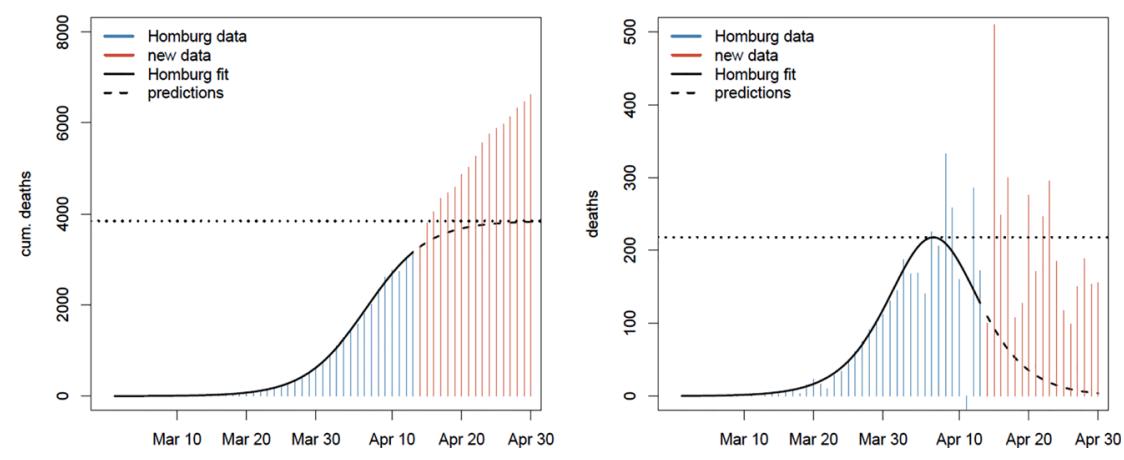

Figure 1: Germany's covid-19 cumulated and daily deaths in March and April 2020.
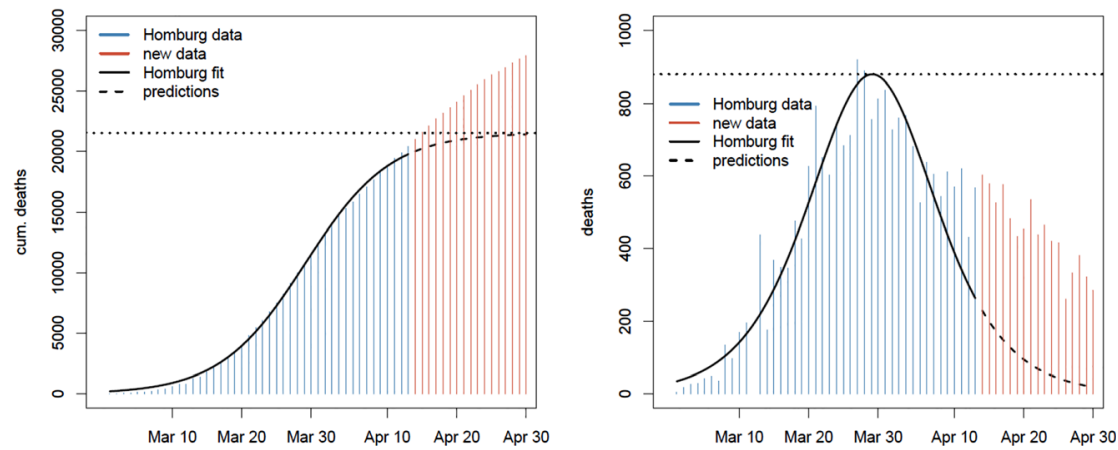

Figure 2: Italy's covid-19 cumulated and daily deaths in March and April 2020. 
Clearly, the curves fitted to the data up to April 13 do not predict the evolution of the epidemic until April 30. Since the epidemic started earlier in Italy, the corresponding model captures the peak of deaths quite accurately. Still, as with Germany, the saturation limit is substantially underestimated. Symmetry of the implied derivatives is also violated in view of the new data, as the latter are substantially skewed to the right.

Moreover, the logistic curve fitted to the observed series of deaths until April 13 (lagged and down-scaled by the lethality) cannot represent the infection trajectory up to the end of the epidemic. For Germany, the curve is truncated seven days after its maximum and its trajectory is affected by the curbing effect of the events after mid-March. The future trajectory to which Homburg had no access will be right skewed, and, thus, not symmetric. Brauer and CastilloChavez (2012), whom Homburg refers to, explicitly caution against the use of logistic functions: "An actual epidemic differs considerably from the idealized models [...] When it is realized that an epidemic has begun, individuals are likely to modify their behavior by avoiding crowds to reduce their contacts and by being more careful about hygiene to reduce the risk that a contact will produce infections" (p. 404). Obviously, estimating the logistic function that features only three parameters and comparing the estimated date of maximum daily deaths with the date of the lockdown in each country is not an adequate research strategy.

\subsection{International Comparisons Do Not Bail Out}

International comparisons cannot compensate for the shortcomings of the data base mentioned above as all countries have been affected. Country-specific differences in the methodology of cause of death certification are not addressed, as well as statements on country-specific differences in the implementation of similarly denominated interventions. Take Switzerland as an example. The "lockdown" measures came into force on March 16, while already being announced on March 13 (and likely having immediate behavioral effects already on that weekend). The measures in Switzerland did not include curfews or bans on gatherings with more than two people as imposed in Germany around March 23 (but bans on more than five). Thus, Swiss "lockdown" measures might resemble more the German measures around March 16 than those around March 23. As with Germany, a longer observation period were needed to test the claim that the lockdown was ineffective. 


\section{What Can Be Said Anyway?}

To begin with, the maximum of deaths in Germany in the JHU dataset, used by Homburg, occurs almost exactly at the same date as the maximum of deaths (8, April) that were reported to and by the Robert Koch Institute (RKI 2020). ${ }^{1}$

More robust than the thin ice of deaths ascribed to Covid-19 appears to be the time trend in excess total mortality. It has long been known that overall mortality is affected by influenza epidemics (Dushoff et al. 2006; Serfling 1963). This should all the more be the case with SARS-CoV-2 featuring a more unfavorable infection kinetics and higher lethality than influenza (Ruan 2020).

The ongoing evolution of deaths compiled by Destatis, the Federal Statistical Office of Germany shows a complex picture. Figure 3 plots the numbers of deceased individuals of age $65+$ who are most likely do succumb to SARS-CoV-2 and to be at risk of avoidable mortality from other causes when medical interventions become temporarily unavailable or are postponed due to the corona crisis. First, a substantial seasonal variability in the number of deaths stands out. In 2017, numbers peaked in calendar week 5. In 2018 a mortality excess with a peak in calendar week 10 occurred. In 2020, the number of deaths in the first four weeks was very similar to that in 2016, 2018 and 2019, but a relative excess can be suspected for calendar weeks 14-15.

If we additionally consider the evolution of reported cases, what Homburg wanted to avoid, a maximum in the reproduction number appears shortly before March 12 (the 4-day moving average on March 11; see Figure 4). The reproduction number published by the RKI lags behind the actual events several days. A peak on March 11 would imply that measures started to be effective before that date (borders closed weekend March 14-15, schools March 16). It is in the nature of an epidemic that the earliest measures can be particularly effective. In airborne or droplet transmission, this would be physical distancing, sneezing etiquette, selfisolation and contact tracing. In the period between March 13 and March 21, the day the reproduction number fell below 1 , the dynamics of the epidemic decelerated. The daily number of reported cases continued to increase, however, reaching its maximum on March 19. Whatever happened around March 12 did not necessarily stop the epidemic, at least not immediately. On April 13, when Homburg submitted his paper, there were 2447 Covid-19 cases reported in German intensive care units, 1841 of them were on ventilators (DIVI 2020).

1 Note that the distribution of COVID-19 deaths by date are part of the German version of the daily report only. 


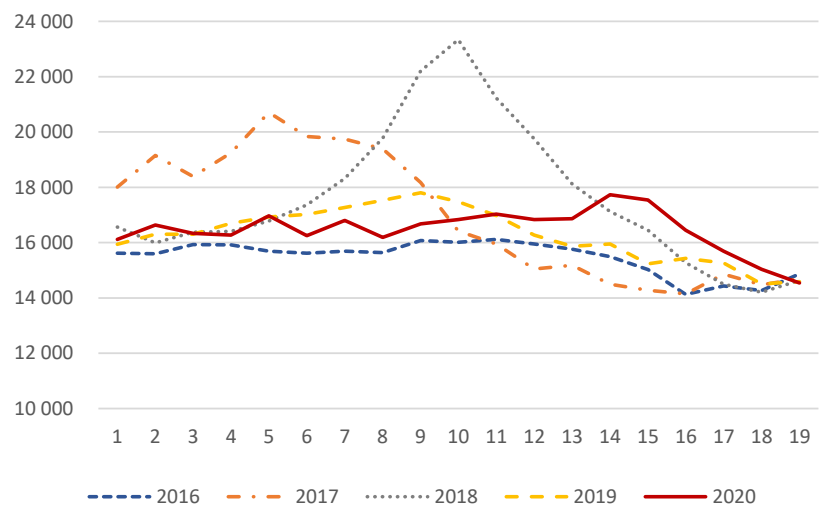

Figure 3: Weekly number of decedents age 65+, Germany, calendar weeks 1-18 from 2016 to 2020, respectively.

Source: Destatis special analysis, May 29, 2020.

\section{The Lockdown's Additional Effect: No Answer}

Homburg posited receding infection rates shortly after March 12, based on two parameters: the peak in the daily evolution of deaths and the time interval of 23 days between infection and death. He does not discuss the robustness of the two parameters. If we include further data such as the (German) excess mortality and

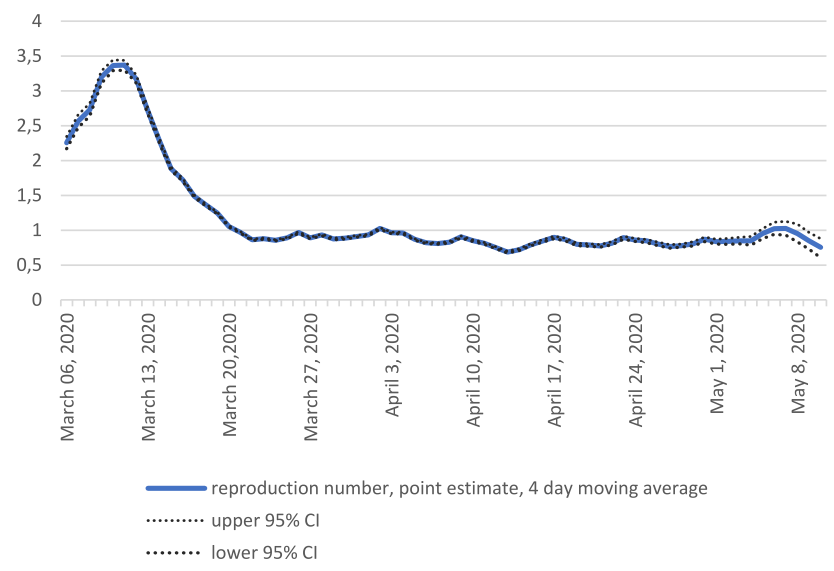

Figure 4: RKl's reproduction number $\mathrm{R}(\mathrm{t})$ and $95 \%$ confidence intervals.

Source: RKI Nowcasting, May 14, 2020. 
the reported SARS-CoV-2 cases, a rather plausible conclusion arises: the measures agreed on March 12, 2020, or already previous preparations, significantly reduced or deferred SARS-CoV-2 infections. If Homburg had presented only that, no objections would arise.

Homburg's quest, however, is for the additional effect of the measures taken at or after March 23, 2020. He should have included all deaths from that day in his 23 day interval, i.e., until April 15, 2020, and, in addition, a sufficiently long period to evaluate the trend. For the assessment of the additional effect, he lacked the data. Furthermore, for his regression model he would have needed additional parameters, to begin with, a variable for marking the date of the presumed lockdown. Such an approach also would have needed to address state-specific heterogeneity, as Bavaria and the City of Freiburg decreed a curfew even before March 23, 2020.

Although explicitly stated otherwise, Homburg failed to conduct an effectiveness analysis of the lockdown - this was not even feasible with his approach. His model is under parameterized and his conclusions, hence, have no empirical basis. They even are misleading as the lockdown is not modelled. More ambitious analyses of the topic have been made available in the meantime. Donsimoni et al. (2020) calibrate a SIR model to Germany, based on infections without symptoms, using data up to April 19 to estimate a counterfactual scenario and forecast future infection rates for each of the 16 federal states. Dehning et al. (2020) also apply a SIR model to Germany, identify change points in the number of infections and correlate them with the dates of individual lockdown measures. Flaxman et al. (2020) and Banholzer et al. (2020) provide pooled cross-country analyses and use different data sources to estimate the impact of governmental interventions on Covid-19 cases. Huber and Langen (2020) assess the impact of German and Swiss containment measures on cumulative covid-19 related hospitalization and death rates. They exploit the fact that the epidemic was more advanced in some regions than in others when measures were implemented. They find that delayed measures come with higher hospitalization and death rates. Contrary to Homburg's claim, all these studies show an impact of different forms of the lockdown on either the number of infections or deaths.

Acknowledgments: We are indebted to Christian Kleiber for help with the fitting of logistic growth curves in Section 2.3 and thank two referees for helpful comments. 


\section{References}

Banholzer, N., E. van Weenen, B. Kratzwald, A. Seeliger, D. Tschernutter, P. Bottrighi, et al. 2020. “Impact of non-pharmaceutical interventions on documented cases of COVID-19." MedRxiv. https://doi.org/10.1101/2020.04.16.20062141.

Betsch, C., L. Korn, L. Felgendreff, S. Eitze, P. Schmid, P. Sprengholz, et al. 2020. “German COVID19 Snapshot Monitoring (COSMO) - Welle 1 (03.03.2020).” PsychArchives 2020: 20200516.

Brauer, F., and C. Castillo-Chavez. 2012. Mathematical Models in Population Biology and Epidemiology, 2nd ed. New York: Springer.

Dehning, J., J. Zierenberg, F. P. Spitzner, M. Wibral, J. P. Neto, M. Wilczek, et al. 2020. “Inferring change points in the spread of COVID-19 reveals the effectiveness of interventions." Science 369(6500), eabb9789. https://doi.org/10.1126/science.abb9789.

DIVI. 2020. Tagesreport 13.04.2020. Deutsche Interdisziplinäre Vereinigung für Intensiv- und Notfallmedizin. https://www.divi.de/divi-intensivregister-tagesreport-archiv.

Donsimoni, J. R., R. Glavion, T. Hartl, et al. 2020. “Covid-19 in Deutschland - Erklärung, Prognose und Einfluss gesundheitspolitischer Maßnahmen.” Perspektiven der Wirtschaftspolitik. https://doi.org/10.1515/pwp-2020-0019.

Dushoff, J., J. B. Plotkin, C. Viboud, D. J. D. Earn, and L. Simonsen. 2006. “Mortality due to influenza in the United States - an annualized regression approach using multiple-cause mortality data." American Journal of Epidemiology 163(2): 181-7.

Flaxman, S., S. Mishra, A. Gandy, H. J. T. Unwin, T. A. Mellan, H. Coupland, et al. 2020. "Estimating the effects of non-pharmaceutical interventions on COVID-19 in Europe." Nature https://doi. org/10.1038/s41586-020-2405-7.

Homburg, S. 2020. "Effectiveness of corona lockdowns: evidence for a number of countries." The Economists' Voice, 20200010. https://doi.org/10.1515/ev-2020-0010.

Huber, M., and H. Langen. 2020. "The impact of response measures on COVID-19-related hospitalization and death rates in Germany and Switzerland.” arXiv: 2005.11278v1. https:// doi.org/10.1093/eurhearti/ehaa291.

Lauer, S. A., K. H. Grantz, Q. Bi, F. K. Jones, Q. Zheng, H. R. Meredith, et al. 2020. “The incubation period of Coronavirus Disease 2019 (COVID-19) from publicly reported confirmed cases: Estimation and application." Annals of Internal Medicine 172(9): 577-82.

RKI. 2020. Täglicher Lagebericht zur Coronavirus-Krankheit-2019 (COVID-19), 12.06.2020 (Coronavirus Disease 2019 (COVID-19), Daily Situation Report of the Robert Koch Institute). Robert Koch Institut.

Ruan, S. 2020. "Likelihood of survival of coronavirus disease 2019." The Lancet Infectious Diseases 20 (6): 630-1.

Serfling, R. E. 1963. "Methods for current statistical analysis of excess pneumonia-influenza deaths." Public Health Reports 78(6): 494-506.

Verity, R., L. C. Okell, I. Dorigatti, P. Winskill, C. Whittaker, N. Imai, et al. 2020. "Estimates of the severity of coronavirus disease 2019: a model-based analysis.” The Lancet Infectious Diseases. https://doi.org/10.1016/S1473-3099(20)30243-7. 